\title{
Cuidados de enfermagem e suas repercussões na vida da pessoa com incontinência anal: revisão integrativa
}

\author{
Nursing care and repercussions in the life of the person with anal incontinence: integrative review \\ Cuidados de enfermería y sus repercusiones en la vida de la persona con incontinencia anal: \\ revisión integrativa
}

\author{
Lívia Nunes Rodrigues Leme'; Norma Valéria Dantas de Oliveira Souza"; Priscilla Farias Chagas ${ }^{\prime \prime \prime}$
}

\begin{abstract}
RESUMO
Objetivo: revisar a produção científica sobre incontinência anal (IA), atualizando o conhecimento de enfermagem sobre o tema. Método: revisão integrativa, realizada em junho/julho de 2018, em bases virtuais de dados e na Revista Estima. Foram selecionados 13 estudos. Resultados: os estudos revisados foram publicados no período de 2006 a 2017, sendo quatro revisões bibliográficas, um estudo prospectivo, dois comparativos descritivos, dois longitudinais, três descritivos e um comparativo. Identificaram-se três categorias analíticas: Tecnologias para o manejo da IA; Principal complicação - dermatite associada à IA e Complexidade dos cuidados de enfermagem e ações terapêuticas. Conclusões: a enfermagem desempenha importante papel na produção científica desta temática, apesar de ainda incipiente. Os cuidados descritos nos estudos revisados repercutem positivamente na vida da pessoa com IA, na medida em que podem prevenir complicações e trazer melhorias na qualidade de vida.
\end{abstract}

Descritores: Incontinência fecal; cuidado de enfermagem; enfermagem; qualidade de vida.

\begin{abstract}
Objective: to review the scientific production on anal incontinence (Al), and nursing knowledge on the subject to date. Method: this integrative review was conducted in June and July 2018 in virtual databases and the journal Revista Estima. Thirteen studies were selected. Results: the studies reviewed, published from 2006 to 2017, comprised four literature reviews and nine reports on other studies: one prospective, two comparative descriptive, two longitudinal, three descriptive and one comparative. Three analytical categories were identified: Technologies for Al management; Main complication - Al-related dermatitis; and Complexity of nursing care and therapeutic actions. Conclusions: knowledge production by nursing on this subject, although still incipient, plays an important role. The care described in the studies has beneficial effects the lives of people with Al, in that it can prevent complications and improve quality of life.

Descriptors: Fecal incontinence; nursing care; nursing; life quality.
\end{abstract}

\section{RESUMEN}

Objetivo: revisar la producción científica sobre incontinencia anal (IA), actualizando los conocimientos de enfermería sobre el tema. Método: revisión integradora, realizada en junio / julio de 2018, en bases de datos virtuales y en el Revista Estima. Se seleccionaron trece estudios. Resultados: los estudios revisados se publicaron de 2006 a 2017, con cuatro revisiones bibliográficas, un estudio prospectivo, dos comparativos descriptivos, dos longitudinales, tres descriptivos y uno comparativo. Se identificaron tres categorías analíticas: Tecnologías para la gestión de la IA; Complicación principal - dermatitis asociada a la IA; y Complejidad del cuidado de enfermería y acciones terapéuticas. Conclusiones: la enfermería desempeña un importante papel en la producción del conocimiento en esta temática, aunque todavía incipiente, visto la captación de sólo 13 artículos, sin recorte temporal. Los cuidados descritos en los estudios son susceptibles de aplicabilidad, repercutiendo positivamente en la vida de la persona con IA en la medida en que pueden prevenir complicaciones y traer mejoras en la calidad de vida. Descriptores: Incontinencia fecal; cuidados de enfermería; enfermería; calidad de vida.

\section{INTRODUÇÃO}

As incontinências são um problema frequente na população, principalmente nos idosos e nas mulheres, resultando em sérios prejuízos biopsicossociais para tal população ${ }^{1,2}$. A incontinência anal (IA) é definida pela Sociedade Internacional de Continência (ICS) como qualquer perda involuntária de fezes e/ou gases, em local inadequado, em qualquer faixa etária, após a obtenção do controle esfincteriano³. 
A continência anal é mantida pela integridade estrutural e funcional da unidade neuromuscular anorretal e do pavimento pélvico ${ }^{4}$. Vários mecanismos contribuem para a continência normal: motilidade intestinal, volume e consistência fecal, nível de consciência, tônus esfincteriano e integridade da inervação neuronal ${ }^{5,6}$. Qualquer patologia que altere esses mecanismos poderá levar a IA, como distúrbios da musculatura esfincteriana anal, do assoalho pélvico, neuromusculares e as alterações da consistência das fezes e da sensibilidade retal ${ }^{7,8}$. A IA é, portanto, um distúrbio que pode ser relacionado à idade e a doenças como Crohn, colite ulcerativa, doença celíaca, diabetes e constipação concomitante e diarréia?

A IA possui prevalência difícil de ser avaliada, devido à subnotificação. As taxas podem variar entre $2 \%$ e $17 \%$ da população geral ${ }^{10}$. No Brasil, existem poucos dados e um estudo cita variações de prevalência que vão desde $3,6 \%$ de incontinência autorreferida até $70,1 \%$, com relato de incontinência fecal líquida ${ }^{11}$.

A subnotificação deve-se a aspectos culturais, constrangimento do paciente ou pela percepção errônea de ser uma consequência normal do envelhecimento ${ }^{11}$. A IA frequentemente leva a distúrbios emocionais e sociais, comprometimento das relações de trabalho, perda da autoestima e depressão ${ }^{2,6,12}$, o que pode gerar situações de maior gravidade.

O tratamento da IA inclui um ou mais dos seguintes itens: dieta, medicamentos, treinamento intestinal, exercícios do assoalho pélvico e biofeedback, cirurgia, irrigação retal, colostomia ${ }^{13}$. O enfermeiro desempenha papel fundamental no cuidado de pessoas com tal distúrbio, aplicando ou orientando vários desses tratamentos, sendo necessário que ele esteja capacitado a desenvolver um cuidado seguro e qualificado a essa clientela. $O$ enfermeiro deve planejar uma assistência individualizada visando o cuidado em suas diferentes dimensões.

É importante conhecer o que a enfermagem tem produzido sobre os cuidados de enfermagem à pessoa com IA e este artigo apresenta como temática o estudo de publicações sobre esses cuidados de enfermagem.

O principal objetivo deste estudo foi revisar a produção científica sobre a IA, atualizando o conhecimento de enfermagem sobre o tema. Selecionaram-se como objetivos específicos: identificar o desenho metodológico de pesquisas relacionadas aos cuidados de enfermagem à pessoa com IA; analisar os cuidados de enfermagem, discutindo suas repercussões na vida da pessoa com IA.

Este estudo justifica-se pela necessidade de aprofundar o conhecimento sobre o tema, com produção dados relevantes que poderão servir de base para futuras pesquisas. Além disso, este trabalho poderá contribuir para a melhoria da qualidade da assistência de enfermagem, visando à prevenção de complicações e à melhor da qualidade de vida do cliente.

\section{Metodologia}

Trata-se de uma pesquisa descritiva, do tipo revisão integrativa, que se caracteriza por proporcionar a síntese de conhecimentos e a incorporação prática de conclusões de estudos reconhecidos cientificamente, devido ao rigor metodológico e achados relevantes ${ }^{14}$. A presente revisão incorpora seis fases.

A primeira foi a de identificação do tema e da questão de pesquisa, qual seja: o que há de publicado sobre os cuidados de enfermagem à pessoa com IA?

Na segunda fase, foram estabelecidos os critérios de inclusão e exclusão dos estudos. Assim, estabeleceram-se como critérios de inclusão: estudos nos idiomas Português, Inglês e Espanhol; de domínio público; sem recorte temporal, para ampliar a captação dos estudos; publicados nas bases de dados Biblioteca Virtual em Saúde (BVS), Scientific Eletronic Library Online (Scielo) e United States National Library of Medicine (PUBMED), além de publicações da Revista Estima, periódico da área de Estomaterapia. Quanto aos critérios de exclusão, foram suprimidas teses, dissertações e monografias.

Os dados foram coletados em junho e julho de 2018, sendo utilizados os seguintes descritores: incontinência fecal e enfermagem, e a palavra-chave incontinência anal, em diferentes combinações, utilizando o boleado AND na pesquisa.

A busca inicial resultou em 3370 estudos, subdivididos em dois grupos, conforme as combinações: incontinência fecal e enfermagem: 2092 estudos; e incontinência anal e enfermagem: 1278 estudos.

Aplicados os critérios de inclusão e exclusão, a busca resultou em 331 artigos, dos quais 51 foram selecionados para leitura completa por se aproximarem do objeto de estudo. Foram excluídos 38 estudos por não atenderem aos objetivos da pesquisa. A amostra final contou com 13 artigos, conforme diagrama apresentado na Figura 1. 


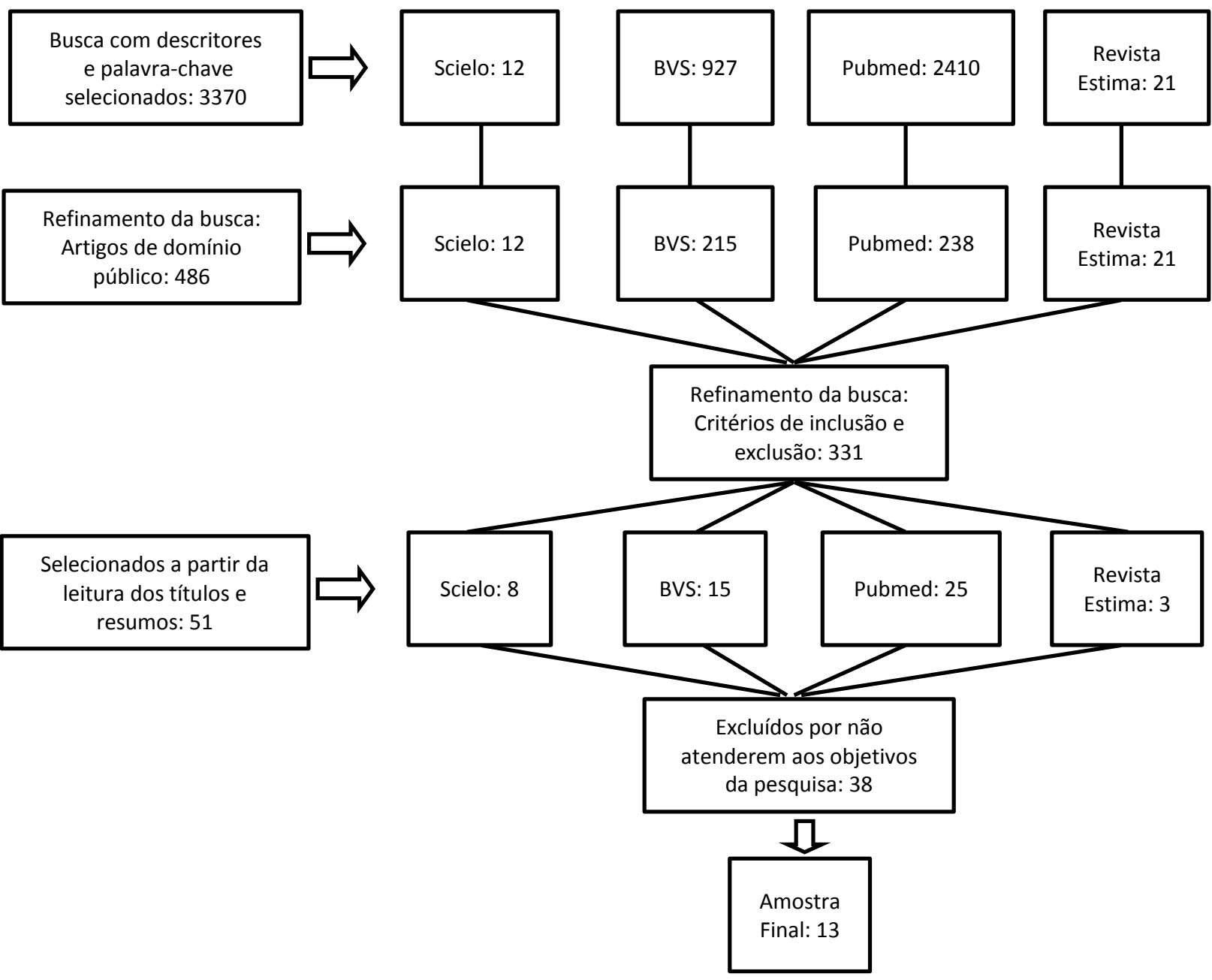

FIGURA 1: Descrição da coleta de dados, 2018.

A terceira fase caracterizou-se pela avaliação, seleção e definição das informações a serem extraídas das publicações captadas. Para tanto, elaborou-se um formulário contendo as seguintes variáveis: título, periódico/Qualis, ano/local de publicação, tipo de estudo e assunto principal e cuidados de enfermagem.

$\mathrm{Na}$ quarta fase, foi realizada a avaliação dos estudos selecionados, com agrupamento em quadro sinóptico que continha informações captadas a partir do formulário descrito. Seguiu-se a quinta fase, da interpretação dos resultados, por meio de análise conjunta do formulário e do quadro sinóptico.

A apresentação dos resultados deu-se por meio de categorias, que emergiram com a lógica de aproximação dos resultados; e seguiu-se a discussão dos achados, compondo a sexta e última fase desta pesquisa. A análise dos estudos permitiu identificar convergências, possibilitando a criação de categorias, que facilitaram o processo de discussão do foco deste estudo. Da análise emergiram três categorias analíticas: Tecnologias para o tratamento da IA; Principal complicação decorrente da IA; e IA e a complexidade de cuidados e ações terapêuticas.

O tratamento dos dados foi realizado à luz da técnica de análise de conteúdo de Bardin. Trata-se de uma técnica de análise que consiste num conjunto de procedimentos em que, por intermédio da interpretação do conteúdo de qualquer classe de documentos, se pode realizar a análise e, com isso, colaborar na interpretação dos resultados ${ }^{15}$.

\section{RESULTADOS E DISCUSSÃO}

O presente estudo revisou 13 artigos ${ }^{4,16-27}$ que atenderam aos critérios previamente estabelecidos e, a seguir, é apresentado um panorama dos mesmos. A caracterização da produção captada está relacionada na Figura 2. 


\begin{tabular}{|c|c|c|c|c|}
\hline Título do artigo & Periódico/Qualis & $\begin{array}{l}\text { Ano/Local de } \\
\text { publicação }\end{array}$ & $\begin{array}{l}\text { Tipo de } \\
\text { estudo }\end{array}$ & Assunto principal \\
\hline $\begin{array}{l}\text { O enfermeiro na atenção à pessoa com } \\
\text { incontinência anal/4. }\end{array}$ & $\begin{array}{l}\text { CuidArte } \\
\text { Enfermagem } \\
\text { B5 }\end{array}$ & $2017 / S P$ & $\begin{array}{l}\text { Revisão } \\
\text { bibliográfica }\end{array}$ & $\begin{array}{l}\text { Atuação do enfermeiro no } \\
\text { cuidado à pessoa com IA }\end{array}$ \\
\hline $\begin{array}{l}\text { Incidence and Characteristics of } \\
\text { Incontinence-Associated Dermatitis in } \\
\text { Community-Dwelling Persons with Fecal } \\
\text { Incontinence }{ }^{16} \text {. }\end{array}$ & $\begin{array}{l}\text { J. Wound Ostomy } \\
\text { Continence Nurs. } \\
\text { A1 }\end{array}$ & 2015/EUA & $\begin{array}{l}\text { Estudo de } \\
\text { coorte } \\
\text { prospectivo }\end{array}$ & $\begin{array}{l}\text { DAl em pacientes com IA não } \\
\text { hospitalizados }\end{array}$ \\
\hline $\begin{array}{l}\text { Nuevos dispositivos de derivación y } \\
\text { colección fecal: efectos adversos } \\
\text { descritos en la bibliografía }{ }^{17} \text {. }\end{array}$ & Enferm. Intensiva & 2014/Espanha & $\begin{array}{c}\text { Revisão } \\
\text { bibliográfica }\end{array}$ & $\begin{array}{l}\text { Equipamentos aplicados para } \\
\text { o controle de fezes }\end{array}$ \\
\hline $\begin{array}{l}\text { Incontinence-associated dermatitis in } \\
\text { community-dwelling individuals with } \\
\text { fecal incontinence }{ }^{18} \text {. }\end{array}$ & $\begin{array}{l}\text { J Wound Ostomy } \\
\text { Continence Nurs. } \\
\qquad \text { A1 }\end{array}$ & 2013/EUA & $\begin{array}{l}\text { Estudo } \\
\text { descritivo e } \\
\text { comparativo }\end{array}$ & $\begin{array}{l}\text { DAl em pacientes com IA não } \\
\text { hospitalizados }\end{array}$ \\
\hline $\begin{array}{l}\text { Profundizando en el conocimiento } \\
\text { sobre el manejo del dispositivo para } \\
\text { control de la eliminación fecal en el } \\
\text { paciente crítico }{ }^{19} \text {. }\end{array}$ & Enferm. Intensiva & 2013/Espanha & $\begin{array}{l}\text { Estudo } \\
\text { observacional } \\
\text { analítico } \\
\text { longitudinal }\end{array}$ & $\begin{array}{l}\text { Equipamentos aplicados para } \\
\text { o controle de fezes }\end{array}$ \\
\hline $\begin{array}{l}\text { Symptoms associated with dietary fiber } \\
\text { supplementation over time in individuals } \\
\text { with fecal incontinence }{ }^{20} \text {. }\end{array}$ & $\begin{array}{l}\text { Nurs. Res. } \\
\quad \text { A1 }\end{array}$ & 2011/EUA & $\begin{array}{l}\text { Estudo } \\
\text { longitudinal }\end{array}$ & $\begin{array}{l}\text { Avaliação da suplementação } \\
\text { de fibras para pacientes com } \\
\text { IA }\end{array}$ \\
\hline $\begin{array}{l}\text { Use and evaluation of disposable } \\
\text { absorbent products for managing fecal } \\
\text { incontinence by community-living } \\
\text { people }{ }^{21} \text {. }\end{array}$ & $\begin{array}{l}\text { J. Wound Ostomy } \\
\text { Continence Nurs. } \\
\text { A1 }\end{array}$ & 2011/EUA & $\begin{array}{c}\text { Análise } \\
\text { descritiva }\end{array}$ & $\begin{array}{l}\text { Uso e avaliação de produtos } \\
\text { absorventes em pacientes } \\
\text { com IA não hospitalizados }\end{array}$ \\
\hline $\begin{array}{l}\text { Effective management of acute fecal } \\
\text { incontinence in hospital: review of } \\
\text { continence management systems }{ }^{22} \text {. }\end{array}$ & $\begin{array}{c}\text { Frontline } \\
\text { Gastroenterol. }\end{array}$ & $\begin{array}{l}\text { 2010/Reino } \\
\text { Unido }\end{array}$ & $\begin{array}{l}\text { Revisão de } \\
\text { literatura }\end{array}$ & $\begin{array}{l}\text { Equipamentos aplicados para } \\
\text { o controle de fezes }\end{array}$ \\
\hline $\begin{array}{l}\text { Diet and eating pattern modifications } \\
\text { used by community-living adults to } \\
\text { manage their fecal incontinence }{ }^{23} \text {. }\end{array}$ & $\begin{array}{l}\text { J. Wound Ostomy } \\
\text { Continence Nurs } \\
\text { A1 }\end{array}$ & 2010/EUA & $\begin{array}{l}\text { Estudo } \\
\text { comparativo } \\
\text { descritivo }\end{array}$ & $\begin{array}{l}\text { Mudanças dietéticas nos } \\
\text { casos de IA }\end{array}$ \\
\hline $\begin{array}{l}\text { Goals of fecal incontinence management } \\
\text { identified by community-living } \\
\text { incontinent adults }{ }^{24} \text {. }\end{array}$ & $\begin{array}{l}\text { West. J. Nurs. Res. } \\
\text { A1 }\end{array}$ & 2010/EUA & $\begin{array}{c}\text { Análise } \\
\text { descritiva }\end{array}$ & $\begin{array}{l}\text { Identificar as metas dos } \\
\text { pacientes com IA }\end{array}$ \\
\hline $\begin{array}{l}\text { Health literacy and emotional responses } \\
\text { related to fecal incontinence }{ }^{25} \text {. }\end{array}$ & $\begin{array}{l}\text { J. Wound Ostomy } \\
\text { Continence Nurs. } \\
\text { A1 }\end{array}$ & 2010/EUA & $\begin{array}{l}\text { Análise } \\
\text { descritiva }\end{array}$ & $\begin{array}{l}\text { Educação em saúde e sua } \\
\text { influência na busca de } \\
\text { cuidados e na comunicação } \\
\text { dos pacientes sobre a IA }\end{array}$ \\
\hline $\begin{array}{l}\text { Comparison of recall and daily self- } \\
\text { report of fecal incontinence severity }{ }^{26} \text {. }\end{array}$ & $\begin{array}{l}\text { J. Wound Ostomy } \\
\text { Continence Nurs. } \\
\text { A1 }\end{array}$ & 2008/EUA & $\begin{array}{c}\text { Análise } \\
\text { comparativa }\end{array}$ & $\begin{array}{l}\text { Comparação entre } \\
\text { recordação e autorrelato } \\
\text { diário da gravidade da IA }\end{array}$ \\
\hline $\begin{array}{l}\text { Fecal incontinence in acutely and } \\
\text { critically ill patients: options in } \\
\text { management }{ }^{27} \text {. }\end{array}$ & $\begin{array}{l}\text { Ostomy Wound } \\
\text { Manage. } \\
\text { B1 }\end{array}$ & 2006/EUA & $\begin{array}{c}\text { Revisão } \\
\text { bibliográfica }\end{array}$ & Opções de manejo da IA \\
\hline
\end{tabular}

FIGURA 2: Produção científica cuidados especializados de enfermagem a pessoas com IA, 2006-2017. Rio de Janeiro,2018.

Os artigos incluídos nesta revisão demostram a importância dos profissionais de enfermagem na produção científica relacionada à temática, ainda que outras áreas tenham também interface com a IA.

Constatou-se que a maior parte dos artigos foi publicada em periódicos de alto impacto científico, sendo oito em periódicos com Qualis $A 1^{16,18,20,21,23-26}$, um em periódico $B 1^{27}$, um em periódico $B 5^{4}$ e em três publicações não foi identificado o Qualis do periódico ${ }^{17,19,22}$. O Qualis é um sistema de avaliação da qualidade da produção intelectual dos 
programas de pós-graduação, numa classificação realizada pelas áreas de avaliação, onde os periódicos são enquadrados em estratos indicativos da qualidade - A1, o mais elevado; A2; B1; B2; B3; B4; B5; C - com peso zero, sendo estes atualizados anualmente ${ }^{28-30}$.

Considerando a importância e o alto rigor científico dos periódicos nos quais a maioria dos artigos captados foi publicada, entende-se que esses estudos tratavam de temas de interesse nacional e internacional, com evidências científicas capazes de auxiliar os profissionais em suas decisões para a melhoria da assistência em diversos aspectos.

Verificou-se que apenas cinco estudos podem ser considerados como mais atuais, com menos de 5 anos de publicação, seis têm entre 5 e 10 anos de publicação e dois foram publicados há mais de 10 anos. Percebe-se, portanto, a necessidade da atualização dos estudos na temática, com vistas à melhoria da produção de conhecimento sobre os cuidados de enfermagem a essa clientela.

Evidenciaram-se, na amostra, quatro revisões bibliográficas, um estudo prospectivo, dois estudos comparativos descritivos, dois estudos longitudinais, três estudos descritivos e um estudo comparativo.

$O$ assunto principal dos artigos captados foi variado, cinco falam sobre equipamentos e produtos para o controle e manejo da IA, dois sobre dermatite associada à incontinência (DAI), dois sobre questões alimentares e dietéticas, um sobre a atuação do enfermeiro frente ao paciente com IA, um sobre identificação de metas dos pacientes com IA, um sobre a influência da educação em saúde e um sobre a comparação entre a recordação e o autorrelato diário da gravidade da IA. A variedade de assuntos revela a amplitude de atuação dos enfermeiros no cuidado à pessoa com IA.

Apresenta-se a seguir as categorias analíticas que emergiram da análise dos estudos captados.

\section{Tecnologias para o manejo da incontinência anal}

Esta categoria discute os equipamentos e adjuvantes utilizados para o manejo e promoção da qualidade de vida de pessoas com IA. Os principais equipamentos citados nos estudos foram aqueles usados para absorção e controle da eliminação das fezes. Os autores citam fraldas ${ }^{27}$, absorventes ${ }^{21}$, sistemas coletores ${ }^{27}$ e de gerenciamento de fezes ${ }^{17,19,22,27}$. Quanto aos adjuvantes, um estudo abordou os produtos de proteção da pele, como cremes ou pomadas de barreira e produtos de limpeza perineal ${ }^{27}$.

As fraldas são equipamentos utilizados para absorver os efluentes do organismo, devendo evitar vazamentos ${ }^{27}$. Seu uso é comum para minimizar os constrangimentos decorrentes da IA. Entretanto, quando usadas de forma inadequada, podem elevar os riscos da ocorrência de lesões cutâneas, como a dermatite perineal ${ }^{4,27}$. Além disso, as fraldas possuem como limitação o não controle do odor. Diante das tecnologias atualmente disponíveis, as fraldas não têm sido consideradas recursos recomendados para o manejo prolongado da IA, devido a esses riscos e limitações ${ }^{27}$.

Os absorventes são frequentemente utilizados por pessoas com IA que estão fora do ambiente hospitalar, a fim de evitar embaraços e reduzir a preocupação e ansiedade relacionadas. Entretanto, poucos produtos absorventes são projetados especificamente para absorver as fezes. Em vez disso, a maioria dos indivíduos deve se conformar usando produtos projetados para a perda de urina ou menstruação ${ }^{21}$. Dessa forma, é necessário que sejam desenvolvidos absorventes específicos para essa clientela, possibilitando o acesso a produtos mais adequados, confortáveis, eficazes e de baixo custo.

Os sistemas coletores consistem em uma barreira de pele autoaderente e uma bolsa anexa que pode ser conectada a um coletor de drenagem à beira do leito. É um sistema fechado, totalmente externo e não invasivo ${ }^{27}$. Quando utilizados de forma adequada, podem prevenir lesões, minimizar o odor, rastrear a saída das fezes com precisão, conservar o fornecimento de cuidados, minimizar gastos e aumentar a aceitação e conforto do paciente ${ }^{27}$.

Os sistemas de gerenciamento consistem em um cateter de silicone mole que é inserido no reto e fixado por meio de balão de retenção de baixa pressão, na extremidade proximal do cateter, destinado a conter e desviar o débito fecal para uma bolsa de coleta, na extremidade distal ${ }^{17,19,27}$. São indicados para pacientes acamados, com fezes líquidas ou semilíquidas ou para pacientes com lesões perineais com risco de infecção ${ }^{17}$.

Esses sistemas possuem diversas vantagens como remoção da umidade na pele e consequente redução de DAI, redução de infecções, promoção de bem-estar e dignidade aos pacientes, redução nos custos de pessoal e de materiais e roupas, possibilidade de irrigação e instilação de medicamento diretamente pelo sistema, caso necessário ${ }^{22,27}$. Alguns efeitos adversos também podem ser citados, como perda de fezes peri sonda, sangramento retal ou anal, devido à necrose ou à ulceração, atonia do esfíncter anal, infecção, obstrução e perfuração intestinal ${ }^{17}$.

Apesar dessas potenciais complicações, os benefícios de tais equipamentos são elevados e seu uso não deve ser desestimulado, recomendando-se inclusive que os indivíduos que façam utilização dos mesmos sejam monitorados rotineiramente para prevenção e identificação precoce de qualquer complicação ${ }^{17,19}$. 
Os produtos adjuvantes, como cremes, pomadas de barreira da pele e produtos de limpeza perineal, são concebidos para prevenir lesões e/ou tratar a pele. São produtos que podem ser combinados com as outras tecnologias, porém com o cuidado de evitar maceração ou de não prejudicar a capacidade adesiva de outros equipamentos, pelo uso dos produtos em excesso ${ }^{27}$.

\section{Principal complicação decorrente da incontinência anal}

A presente categoria discorre sobre a principal complicação da IA citada nos estudos, a dermatite associada à incontinência (DAI), uma inflamação da pele associada à umidade, em consequência do seu contato com as fezes, no caso da IA, ou ainda com fezes e urina, na incontinência mista ${ }^{4,16,18}$. Apresenta-se com áreas de eritema, edema ou com perda da integridade da pele, as quais podem causar desconforto e/ou dor. Indivíduos com DAI também apresentam maior risco de desenvolver lesões por pressão na região afetada. Os danos cutâneos da DAI podem ocorrer em várias áreas do períneo, região interglútea, coxas e área da virilha ${ }^{16,18}$. As enzimas e bactérias intestinais podem afetar a integridade da pele e causar os danos descritos.

Os efeitos secundários do contato da pele com as fezes são a hiper-hidratação e a maceração, bem como a elevação da temperatura na região, em razão do uso inadequado de dispositivos de contenção, como fraldas e absorventes ${ }^{4}$.

A prevalência de DAI pode chegar a cerca de $20 \%$ em pacientes hospitalizados, $50 \%$ em pacientes criticamente enfermos e $41 \%$ em pacientes com IA não hospitalizados ${ }^{16}$. Nesses últimos, a gravidade da DAl é tipicamente leve, com disseminação geralmente limitada a apenas uma área do corpo, sendo o eritema o sintoma mais comum. Apesar dos sintomas leves, a DAI frequentemente produz desconforto, com relatas de dor entre $78 \%$ e $38 \%$ dos indivíduos, apresentando também sintomas de prurido ou queimação ${ }^{16}$.

A associação entre a gravidade da IA e a gravidade da DAI é fraca, sugerindo que a pessoa com IA de qualquer gravidade possui risco aumentado de DAI. As características específicas da gravidade da IA que são significativamente relacionadas com a gravidade da DAI são a quantidade de fezes vazadas e a frequência de incontinência ${ }^{18}$.

O tempo para o início dos sintomas da DAl em indivíduos não hospitalizados varia em aproximadamente 14 dias, o que é consideravelmente longo quando comparado a pacientes críticos e gravemente enfermos, quando o tempo médio para início dos sintomas é de 4 dias $^{16}$.

A consistência das fezes pode ou não implicar maior risco para a DAI. Um estudo cita que o contato prolongado da pele com fezes de qualquer tipo de consistência representa risco aumentado ${ }^{18}$, enquanto outro menciona que as fezes líquidas estão associadas à maior probabilidade de DAl, devido ao maior teor de irritantes para a pele, má absorção de nutrientes e comprometimento da nutrição do paciente, principalmente nos hospitalizados ${ }^{4}$.

\section{Incontinência anal e a complexidade de cuidados e ações terapêuticas}

Esta categoria busca discutir a complexidade dos cuidados e ações terapêuticas adotadas para curar e/ou para minimizar a problemática da IA, inclusive, destacando-se a apropriação de conhecimentos de outras áreas como, por exemplo, nutrição e fisioterapia, com o fito de promover a melhoria da qualidade de vida a pessoas com IA.

Os cuidados de enfermagem a essa clientela iniciam-se com a questão da educação em saúde e a orientação nos diversos aspectos que envolvem a IA e o alcance do bem-estar. Os estudos que fazem parte desta revisão citam que os

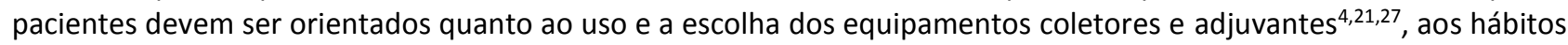
alimentares e às mudanças dietéticas para melhoria da $I A^{4,20,23}$, aos medicamentos que podem influenciar o surgimento e agravamento da $\mathrm{IA}^{4}$, aos cuidados habituais com a pele ${ }^{16} \mathrm{e}$, ainda, quanto aos métodos de prevenção da $\mathrm{DAl} \mathrm{I}^{4,16,18}$.

A avaliação das modalidades terapêuticas também faz parte dos cuidados de enfermagem, de forma a selecionar junto ao paciente o melhor recurso terapêutico para cada caso. 0 enfermeiro deve avaliar e orientar os exercícios do assoalho pélvico, biofeedback, irrigação, estimulação percutânea do nervo tibial e técnicas cognitivas para diminuir a ansiedade ${ }^{4}$. A identificação das opções de gerenciamento para IA, que são preferidas pelos pacientes, permitem um melhor planejamento de acordo com prioridades e preferências dos mesmos, estabelecendo assim, maior probabilidade de adesão aos tratamentos propostos ${ }^{24}$.

Em relação às tecnologias para o manejo da IA, diversos são os cuidados que o enfermeiro deve desenvolver. As fraldas e demais equipamentos absorventes precisam ser trocados a cada episódio de IA, promovendo a limpeza da pele e o uso de cremes barreira, a fim de prevenir a ocorrência da $D A I^{4,27}$. Os cuidados com os sistemas de gerenciamento fecal também necessitam fazer parte da rotina laboral do enfermeiro ${ }^{19,22,27}$. É recomendado, por exemplo, a identificação do perfil de pessoas em risco de complicações $^{19}$, o exame digital antes da inserção do sistema, manejo das complicações, como obstruções e desvio, além do controle do balão de retenção ${ }^{22}$. 
O enfermeiro deve ainda investigar a DAI em pacientes não hospitalizados, questionando-os sobre a IA, quanto à presença de sintomas e à gravidade, já que o estigma e o constrangimento decorrentes da mesma, muitas vezes, impedem que os pacientes busquem ajuda espontaneamente ${ }^{16,18}$. O enfermeiro deve proceder o ensino da autoavaliação, com o uso de espelhos, por exemplo, para verificar a região perineal e adjacências ${ }^{16}$. Pacientes hospitalizados e em uso de tecnologias para o manejo da IA também precisam ser avaliados rotineiramente quanto à prevenção e cuidados da DAl ${ }^{17,19,22,26}$.

O apoio emocional, igualmente, faz parte dos cuidados de enfermagem a essa clientela. O profissional deve aconselhar e apoiar a pessoa a superar os contratempos e a refletir sobre seus próprios objetivos, além de conhecer as possíveis respostas emocionais à IA a fim de promover apoio terapêutico, facilitando a aceitação da IA e melhor adesão ao tratamento ${ }^{24,25}$.

\section{CONCLUSÃO}

O estudo revela a importância da enfermagem na produção do conhecimento nesta temática, porém ainda é incipiente, haja vista a captação de somente 13 artigos, sem determinação de recortes temporal, obtendo-se publicações de 2006 a 2017.

A enfermagem está diretamente ligada aos diversos tipos de cuidados descritos, os quais podem repercutir positivamente na vida da pessoa com IA, na medida em que podem prevenir complicações e trazer melhorias na qualidade de vida da clientela assistida. O enfermeiro deve promover uma abordagem individualizada e abrangente de forma a possibilitar maior adesão aos tratamentos propostos, resultando em melhor prognóstico.

Considera-se que uma limitação do estudo foi a adoção de análise de artigos apenas de domínio público, o que restringiu a captação de um maior quantitativo de publicações, as quais poderiam enriquecer ainda mais a produção de resultados e a discussão dos dados coletados.

\section{REFERÊNCIAS}

1. Gonçalves FGA, Reis FLM, Silva NAB, Souza NVDO, Varella TCMML, Pires AS. Stomatherapy content and teaching strategies in the undergraduate nursing curriculum. Rev. enferm. UERJ, 2018 [cited 2019 Jul 24]; 26:e28921. DOI: http://dx.doi.org/10.12957/reuerj.2018.28921

2. Faria CA, Benayon PC, Ferreira ALV. Prevalence of anal and double incontinence in elderly women and impact on their quality of life. Arq. ciênc. saúde. 2018 [cited 2019 Jul 24]; 25(1) 41-5. DOI: http://dx.doi.org/10.17696/2318-3691.25.1.2018.865

3. Abrams P, Andersson L, Birder L. Fourth International Consultation on Incontinence. Recommendations of the International Scientific Committee: evaluation and treatment of urinary incontinence, pelvic organ prolapsed and fecal incontinence. Neurourol. Urodyn. 2010 [cited 2018 Jun 01]; 29 (1):213-240. Avaliable from: http://onlinelibrary.wiley.com/doi/10.1002/nau.20870/epdf

4. Silveira BL, Catarucci F, Macedo GM, Cunha LD, Romano VC, Gomes JJ. The nurse in attention to the person with anal incontinence. CuidArte, Enferm. 2017 [cited 2018 Jun 01]; 11(2): 215-22. Avaliable from: http://pesquisa.bvsalud.org/brasil/resource/pt/bde-32949

5. Leite J, Poças F. Tratamento da incontinência fecal. Rev. port. coloproct. 2010 [cited 2018 Jun 01]; 7(2): 68-72. Avaliable from: http://www.spcoloprocto.org/uploads/recomendac807_0771_es_tratamento_da_incontine770_ncia_fecal.pdf

6. Azevedo M, Guilhem DB, Hobo TMW, Goulart MV. Avaliação da predominância da incontinência anal nos partos vaginal e cesáreo. Universitas: Ciências da Saúde. 2017 [cited 2018 Jun 01]; 15(2): 101-6. DOI: http://dx.doi.org/10.5102/ucs.v15i2.4240

7. Quintão MG, Oliveira SAS, Guedes HM. Fecal incontinence: profile of old residents in the city of Rio Piracicaba, state of Minas Gerais, Brazil. Rev. bras. geriatr. Gerontol. 2010 [cited 2018 Jun 01]; 13(2) 191-201. Avaliable from: http://www.scielo.br/pdf/rbgg/v13n2/a04v13n2.pdf

8. Ribeiro FSL. Incontinência fecal: abordagem passo a passo [master thesis]. Universidade do Porto; Porto (Pt) 2013. [cited 2018 Jun 01] Avaliable from: https://repositorio-aberto.up.pt/bitstream/10216/71769/2/30712.pdf

9. Menees SB, Almario CV, Spiegel BMR, Chey WD. Prevalence of and factors associated with fecal incontinence: results from a population-based survey. Gastroenterology. 2018 [cited 2018 Jul 01]; 154(6): 1672-1681.e3. DOI: http://dx.doi.org/10.1053/j.gastro.2018.01.062

10. Kaiser Junior RL, Silva G, Braile DM, Wexner SD. Influence of sphincter defect on biofeedback outcomes in patients with fecal incontinence. J. coloproctol. [Internet]. 2014. [cited 2018 Jun 01]; 34(2): 67-72. DOI: http://dx.doi.org/10.1016/j.jcol.2014.04.004

11. Santos CRS, Lima TGS, Schmidt FMQ; Santos VLCG. Pelvic floor rehabilitation in anal incontinence In: Catto-Smith AG. Fecal incontinence: causes, management and outcome. Croácia: IntechOpen; 2014. p.107-22. [ cited 2018 Jul 10] Avaliable from: https://www.intechopen.com/books/fecal-incontinence-causes-management-and-outcome/pelvic-floor-rehabilitation-in-analincontinence

12. Rodrigues BDS, Reis IGN, Coelho FMO, Buzatti KCLR. Fecal incontinence and quality of life assessment through questionnaires. J. coloproctol.[Internet]. 2017 [cited 2018 Jun 01]; 37(4): 341-8. DOI http://dx.doi.org/10.1016/j.jcol.2017.06.008. 
13. Arzu I. Fecal incontinence. In: Catto-Smith AG. Fecal incontinence: causes, management and outcome. Croácia: IntechOpen; 2014. p.15-30.[ cited 2018 Jun 01] Avaliable from: https://www.intechopen.com/books/fecal-incontinence-causesmanagement-and-outcome/fecal-incontinence

14. Souza MT, Silva MD, Carvalho R. Revisão integrativa: o que é e como fazer. Einstein [LILACS-Sistema Latino-Americano e do Caribe de Informação em Ciências da Saúde] 2010 [cited 2018 Jun 01]; 8(1):102-5. Avaliable from: www.scielo.br/pdf/eins/v8n1/pt_1679-4508-eins-8-1-0102.pdf

15. Bardin, L. Análise de conteúdo. Lisboa (Pt): Edições 70; 2011.

16. Bliss DZ, Funk T, Jacobson M, Savik K. Incidence and characteristics of incontinence-associated dermatitis in communitydwelling persons with fecal incontinence. J. wound ostomy continence nurs. 2015 [cited 2018 Jul 01]; 42(5): 525-30. DOI: http://dx.doi.org/10.1097/WON.0000000000000159

17. Sánchez López MI. New devices of fecal diversion and collection: adverse effects described in the literature. Enferm. Intensiva 2014 [cited 2018 Jul 01]; 25(1): 4-14. DOI: http://dx.doi.org/10.1016/j.enfi.2013.11.001

18. Rohwer K, Bliss DZ, Savik K. Incontinence-associated dermatitis in community-dwelling individuals with fecal incontinence. J. wound ostomy continence nurs. 2013 [cited 2018 Jun 01]; 40(2): 181-4. DOI: http://dx.doi.org/10.1097/WON.0b013e31827e8b3d

19. Mera MJF, Díez LV, Gaute NF, Martín AIC, Gil DM, Miralles MJF, et al. To go in-depth in the knowledge of the device to control fecal elimination in the critical patient. Enferm. Intensiva. 2013 [cited 2018 Jun 01]; 24(1): 23-35. DOI: http://dx.doi.org/10.1016/j.enfi.2012.11.001.

20. Bliss DZ, Savik K, Jung HJ, Whitebird R, Lowry A. Symptoms associated with dietary fiber supplementation over time in individuals with fecal incontinence. Nurs. Res. 2011 [cited 2018 Jun 01]; 60(3 Suppl):S58-67. DOI: http://dx.doi.org/10.1097/NNR.0b013e3182186d8c

21. Bliss DZ, Lewis J, Hasselman K, Savik K, Lowry A, Whitebird R. Use and evaluation of disposable absorbent products for managing fecal incontinence by community-living people. J. wound ostomy continence nurs. 2011 [cited 2018 Jun 01]; 38(3):289-97. DOI: http://dx.doi.org/10.1097/WON.0b013e31821530ca

22. Ousey K, Gillibrand W, Lui S. Effective management of acute faecal incontinence in hospital: review of continence management systems. Frontline gastroenterol. 2010 [cited 2018 Jun 01];1(2):94-97. DOI: http://dx.doi.org/10.1136/fg.2009.001206

23. Croswell E, Bliss DZ, Savik K. Diet and eating pattern modifications used by community-living adults to manage their fecal incontinence. J. wound ostomy continence nurs. 2010 [cited 2018 Jun 01]; 37(6):677-82. DOI: http://dx.doi.org/10.1097/WON.0b013e3181feb017

24. Manthey A, Bliss DZ, Savik K, Lowry A, Whitebird R. Goals of fecal incontinence management identified by community-living incontinent adults. West. j. nursing res. 2010 [cited 2018 Jun 01]; 32(5):644-61. DOI: http://dx.doi.org/10.1177/0193945909356098

25. Patel K, Bliss DZ, Savik K. Health literacy and emotional responses related to fecal incontinence. J. wound ostomy continence nurs. 2010 [cited 2018 Jun 01]; 37(1):73-9. DOI: http://dx.doi.org/10.1097/WON.0b013e3181c68e7b

26. Fisher K, Bliss DZ, Savik K. Comparison of recall and daily self-report of fecal incontinence severity. J. wound ostomy continence nurs. 2008 [cited 2018 Jun 01]; 35(5): 515-20. Avaliable from: https://www.ncbi.nlm.nih.gov/pmc/articles/PMC5250620/

27. Beitz JM. Fecal incontinence in acutely and critically ill patients: options in management. Ostomy wound Manage. 2006 [cited 2018 Jun 01]; 52(12): 56-8, 60, 62-6. Avaliable from: https://www.o-wm.com/content/fecal-incontinence-acutely-and-criticallyill-patients-options-management

28. Fundação Capes [site de Internet]. Qualis. 2014 [cited 2018 Jul 01]. Avaliable from http://www.capes.gov.br/sala-deimprensa/noticias/2550-capes-aprova-a-nova-classificacao-do-qualis.

29. Barata, RCB. Dez coisas que você deveria saber sobre o Qualis. RBPG, 2016 [cited 2018 Jul 01]; v.13, n.1. Avaliable from https://pos.letras.ufg.br/up/26/o/artigo_10_coisas.pdf

30. Erdmann AL, Marziale MHP, Pedreira MLG, Lana FCF, Pagliuca LMF, Padilha MI et al. Evaluation of scientific periodicals and the brazilian production of nursing articles. Rev. latinoam. enferm. (Online) 2009 [cited $2018 \mathrm{Jul}$ 01]; 17(3). Avaliable from: https://www.redalyc.org/pdf/2814/281421908019.pdf 